Article

\title{
A Critical Appraisal of National and International Clinical Practice Guidelines Reporting Nutritional Recommendations for Age-Related Macular Degeneration: Are Recommendations Evidence-Based?
}

\author{
John G. Lawrenson ${ }^{1} * \mathbb{D}$, Jennifer R. Evans ${ }^{2}$ and Laura E. Downie ${ }^{3}(\mathbb{D}$ \\ 1 Division of Optometry and Visual Science, City, University of London, Northampton Square, \\ London EC1V OHB, UK \\ 2 International Centre for Eye Health, Clinical Research Department, London School of Hygiene and Tropical \\ Medicine, Keppel St, London WC1E 7HT, UK; Jennifer.Evans@lshtm.ac.uk \\ 3 Department of Optometry and Vision Sciences, The University of Melbourne, Parkville, Victoria 3010, \\ Australia; ldownie@unimelb.edu.au \\ * Correspondence: j.g.lawrenson@city.ac.uk; Tel.: +44(0)20-7040-4310
}

Received: 26 March 2019; Accepted: 9 April 2019; Published: 11 April 2019

check for updates

\begin{abstract}
Eye care professionals should have access to high quality clinical practice guidelines that ideally are underpinned by evidence from robust systematic reviews of relevant research. The aim of this study was to identify clinical guidelines with recommendations pertaining to dietary modification and/or nutritional supplementation for age-related macular degeneration (AMD), and to evaluate the overall quality of the guidelines using the Appraisal of Guidelines for Research and Evaluation II (AGREE II) instrument. We also mapped recommendations to existing systematic review evidence. A comprehensive search was undertaken using bibliographic databases and other electronic resources for eligible guidelines. Quality appraisal was undertaken to generate scores for each of the six AGREE II domains, and mapping of extracted nutritional recommendations was performed for systematic reviews published up to March 2017. We identified 13 national and international guidelines, developed or updated between 2004 and 2019. These varied substantially in quality. The lowest scoring AGREE II domains were for 'Rigour of Development', 'Applicability' (which measures implementation strategies to improve uptake of recommendations), and 'Editorial Independence'. Only four guidelines used evidence from systematic reviews to support their nutritional recommendations. In conclusion, there is significant scope for improving current Clinical Practice Guidelines for AMD, and guideline developers should use evidence from existing high quality systematic reviews to inform clinical recommendations.
\end{abstract}

Keywords: clinical practice guidelines; systematic reviews; age-related macular degeneration; nutritional supplements; diet; nutrition; AGREE II

\section{Introduction}

Age-related macular degeneration (AMD) is an ocular disease affecting the central area of the retina (the macula), which is responsible for high-resolution daytime vision [1]. In the early stages of AMD, the macula shows characteristic sub-retinal lipoprotein deposits, known as drusen, and the monolayer of pigmented cells beneath the retina (retinal pigment epithelium) shows areas of hypo- and hyper-pigmentation. As the disease progresses, the retinal pigment epithelium can become atrophic, with secondary dysfunction and a loss of retinal photoreceptors [2]. Less commonly, new blood vessels grow beneath, or within, the retina; these vessels have a tendency to leak, causing disruption to 
the macular architecture and ultimately scar formation (neovascular AMD). Early AMD is typically asymptomatic; however, later stages of AMD can have a significant negative impact on visual function and quality of life [3]. AMD is currently the leading cause of severe vision impairment among people aged 50 and over in high-income countries [4]. With increasing life expectancy these numbers are projected to increase substantially in the future.

AMD is a complex disorder with several non-modifiable and modifiable risk factors [5]. The retina is particularly susceptible to oxidative stress, as a result of its high oxygen consumption and exposure to light; animal and cell culture studies have identified oxidative stress as a contributory factor for the development of AMD [6]. A large body of observational and experimental research in humans has investigated the association between dietary antioxidants and AMD, in particular whether an increased intake of antioxidant vitamin and minerals or specific carotenoids can prevent or slow the progression of the disease $[7,8]$. The role of particular essential fatty acids has also been investigated, including exploiting the anti-inflammatory properties of long-chain omega-3 fatty acids.

Based on accumulating research evidence, dietary modification and/or nutritional supplementation has been proposed as a simple and potentially cost-effective strategy for modifying the risk of AMD. Although there is a large body of research in this area, the quality of studies is highly variable and conflicting results have been reported. These factors pose challenges for clinicians, when attempting to provide evidence-based recommendations to patients about the relative risks and benefits of nutrition-based interventions. Clinical practice guidelines can be used to aid clinical decision-making in such circumstances. These documents should contain recommendations that are informed by a systematic review of the available research evidence, and a consideration of the benefits and harms of alternative interventions [9]. In the process of guideline development, developers either elect to perform their own systematic review of the literature, or to instead integrate the results from previously published reviews. Systematic reviews involve a process of systematically identifying, appraising and synthesising findings from all relevant research studies relating to a particular research question, with the intent of minimising the potential for bias. The objectives of the present study were to:

(1) Identify clinical practice guidelines for AMD that contain nutritional recommendations, based upon a systematic literature search;

(2) Evaluate the quality of these guidelines using the Appraisal of Guidelines for Research and Evaluation II (AGREE II) tool; and

(3) Map clinical practice guideline recommendations, as related to nutrition and AMD, to relevant systematic reviews.

\section{Materials and Methods}

\subsection{Eligibility Criteria}

We included national and international clinical practice guidelines for AMD that contained nutritional recommendations, including dietary modification or nutritional supplementation, as strategies to prevent or slow the progression of the disease.

\subsection{Search Strategy}

We searched the Ovid MEDLINE and Embase bibliographic databases from January 1999 to January 2019 using search filters for identifying clinical guidelines that were developed by the Canadian Agency for Drugs and Technologies in Health (https://www.cadth.ca) (see Supplementary Materials). In addition to the bibliographic database searches, we also searched Guideline Central Summaries (https://www.guidelinecentral.com/summaries/) and the Turning Research into Practice (TRIP) database (https://www.tripdatabase.com/), and undertook a search of the webpages of national and international professional organisations for ophthalmology and optometry. The search was not limited by language and we used Google Translate to extract recommendations and appraise guidelines that were not written in English. 


\subsection{Study Selection and Data Extraction}

Following removal of duplicates, two reviewers (JL/LD) independently screened the titles and abstracts identified from the bibliographic searches and resolved any discrepancies by discussion and consensus. We obtained full-text copies of potentially eligible guidelines and these were independently assessed by all three authors, working in pairs, to determine whether they met the inclusion criteria. Reasons for exclusion were documented at this stage. Two reviewers (all three authors working in pairs) independently extracted general characteristics of each guideline (e.g., author/organisation, country, year of publication, target audience) and details of the specific nutritional recommendations.

\subsection{Appraisal of Clinical Guidelines}

The quality of each clinical guideline was independently evaluated by two reviewers (all three authors working in pairs) using the Appraisal of Guidelines for Research and Evaluation (AGREE) II tool (https://www.agreetrust.org/). The AGREE II instrument evaluates the process of practice guideline development, and the quality of reporting, using 21 items organised into six key domains, as follows: (1) Scope and Purpose; (2) Stakeholder Involvement; (3) Rigour of Development; (4) Clarity of Presentation; (5) Applicability; and (6) Editorial Independence. Table 1 provides further details about the content of these domains. Each item within the AGREE II tool comprises a quality statement/concept, which is scored using a 7-point Likert rating scale. A score of ' 1 ' was given when there was no information that was relevant to the item or where the concept was very poorly reported. A score of ' 7 ' was given if the quality of reporting met the full criteria, as defined in the AGREE II User's Manual. The overall scores for each of the six domains were calculated by summing up all the scores of the individual items for that domain, and then scaling the total as a percentage of the maximum possible score for each domain. Therefore higher scores indicate higher guideline quality. An intra-class correlation coefficient (ICC) was used to evaluate overall inter-rater agreement.

Table 1. Clinical guideline quality domains used in the Appraisal of Guidelines for Research and Evaluation II (AGREE II) tool.

\begin{tabular}{lr}
\hline \multicolumn{1}{c}{ Domain } & Content \\
\hline $\begin{array}{l}\text { Scope and Purpose (3 items) } \\
\text { Stakeholder Involvement (3 items) }\end{array}$ & $\begin{array}{r}\text { Focuses on the extent to which the guideline was developed by the appropriate } \\
\text { stakeholders and represents the views of its intended users }\end{array}$ \\
\hline Rigour of Development ( 8 items) & $\begin{array}{r}\text { Relates to the process used to gather and synthesize the evidence, the methods } \\
\text { to formulate the recommendations, and to update them }\end{array}$ \\
\hline Clarity of Presentation (3 items) & $\begin{array}{r}\text { Deals with the language, structure, and format of the guideline } \\
\text { Applicability (4 items) }\end{array}$ \\
\hline Editorial Independence (2 items) & $\begin{array}{r}\text { Pertains to the likely barriers and facilitators to implementation, strategies to } \\
\text { improve uptake, and resource implications of applying the guideline }\end{array}$ \\
\hline
\end{tabular}

\subsection{Mapping Clinical Guideline Recommendations to Systematic Review Evidence}

Given the predominance of systematic reviews in the hierarchy of clinical evidence [10], we undertook an exercise to map the extracted guideline recommendations relating to diet or nutritional supplementation, in the context of AMD, to the evidence derived from systematic reviews. We also assessed whether recommendations were linked to particular citations (e.g., systematic reviews, individual randomised controlled trials (RCTs), or other study designs). To identify relevant systematic reviews on nutritional interventions for AMD we used two recently published studies $[11,12]$ that identified and critically appraised systematic reviews of AMD interventions. Lindsley et al. [11] identified 47 systematic reviews published between 2001 and 2014, of which 9 (19\%) evaluated dietary supplements. A more recent study by Downie and colleagues [12] found 71 systematic reviews 
published between 2003 and 2017, with 10 (12\%) relating to nutritional interventions in AMD. These studies identified 11 unique reviews [13-23], of which over 50\% were published after 2014.

The process of mapping systematic reviews to guideline recommendations relating to nutrition was performed by a single reviewer and then independently checked by a second reviewer.

\section{Results}

\subsection{Search Results}

The searches identified 868 potentially relevant records. Following title and abstract screening, 838 were excluded. After a full-text review of 30 potentially eligible records, 17 were excluded as they did not contain recommendations for nutritional interventions. Thirteen guidelines were included in the final analysis. Figure 1 shows the flow diagram for guideline selection.

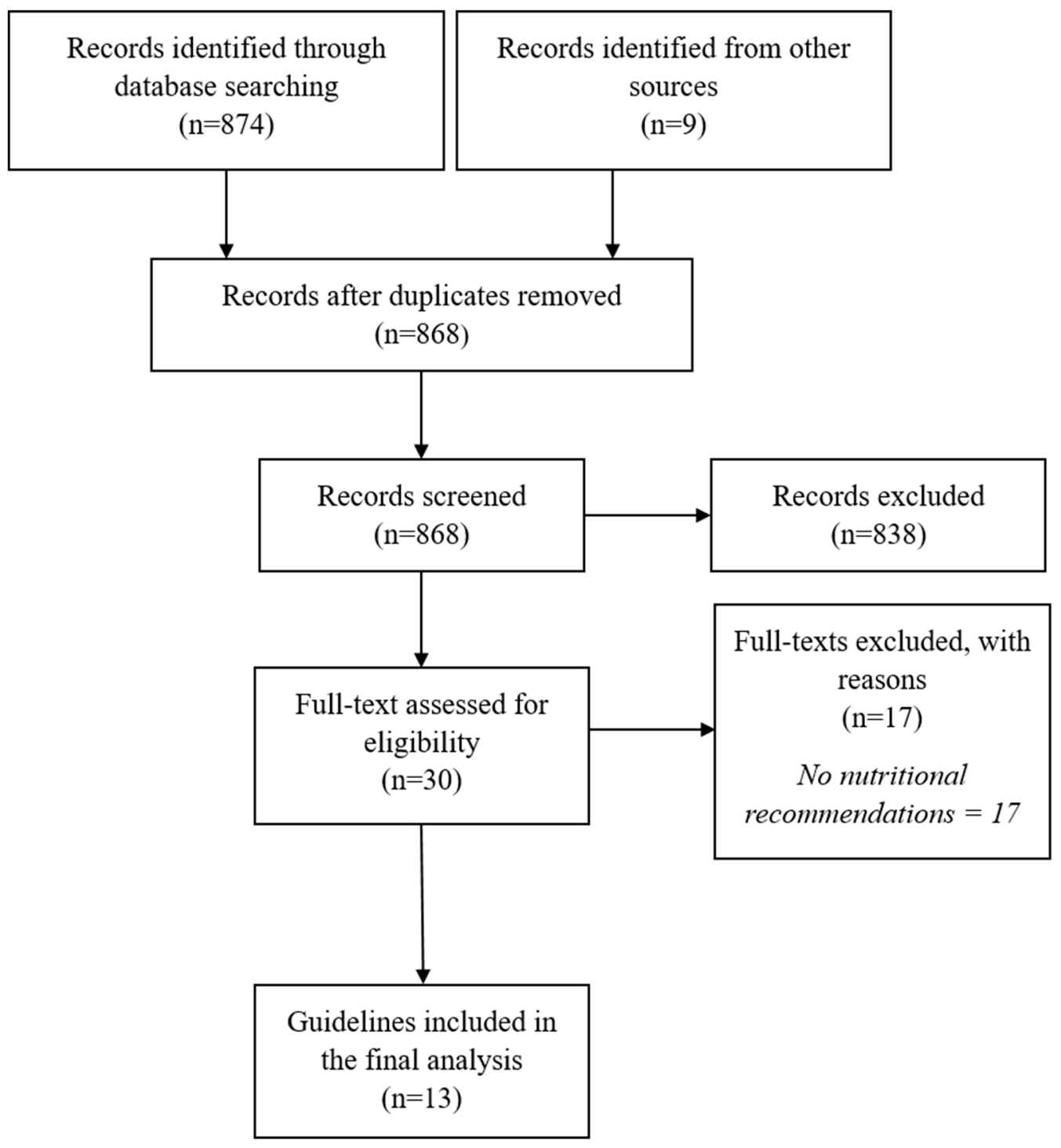

Figure 1. Selection process for identifying relevant clinical practice guidelines.

\subsection{Characteristics of Included Guidelines and Nutritional Recommendations}

Table 2 summarises the characteristics of 13 national guidelines from the United Kingdom [24,25], United States [26-28], Canada [29,30], Australia [31], New Zealand [32], Spain [33], Germany [34], and the Philippines [35], and an international ophthalmology guideline [36], that were published between 
2004 and 2019. Three guidelines were produced by government organisations [24,25,32], while the remainder were developed by professional societies or associations for specific eye care professions.

Table 2. General characteristics of the included age-related macular degeneration (AMD) clinical practice guidelines.

\begin{tabular}{lcccc}
\hline \multicolumn{1}{c}{ Organisation } & Reference & Year & Country & Target Audience \\
\hline American Optical Association & {$[26]$} & 2004 & United States & Optometrists \\
\hline International Council of Ophthalmology & {$[36]$} & 2007 & International & Optometrists \\
\hline Spanish Retina and Vitreous Society (SERV) & {$[33]$} & 2009 & Spain & Ophthalmologists \\
\hline Canadian Expert Consensus & {$[30]$} & 2012 & Canada & Ophthalmologists \\
\hline German Ophthalmological Society (in German) & {$[34]$} & 2014 & Germany & Ophthalmologists \\
\hline Eye Health Council of Ontario & {$[29]$} & 2015 & Canada & Health and eye care professionals \\
\hline National Health Committee & {$[32]$} & 2015 & New Zealand & $\begin{array}{c}\text { Healthcare professionals involved in the } \\
\text { diagnosis and management of AMD }\end{array}$ \\
\hline American Academy of Ophthalmology & {$[27]$} & 2015 & United States & Ophthalmologists \\
\hline Vitreo-retina Society of the Philippines (VRSP) & {$[35]$} & 2016 & Philippines & Ophthalmologists \\
\hline $\begin{array}{l}\text { National Institute for Health and Care Excellence } \\
\text { (NICE; Clinical Knowledge Summaries) }\end{array}$ & {$[24]$} & 2016 & United Kingdom & Primary care healthcare professionals \\
\hline Clinical Advisory Committee [28] & {$[28]$} & 2017 & United States & Optometrists \\
\hline $\begin{array}{l}\text { NICE (NG82) } \\
\text { Optometry Australia }\end{array}$ & {$[25]$} & 2018 & United Kingdom & $\begin{array}{c}\text { Healthcare professionals involved in the } \\
\text { diagnosis and management of AMD }\end{array}$ \\
\hline
\end{tabular}

Eight guidelines [24,26-29,31,34,35] provided nutritional recommendations (either positive or negative) for reducing the risk of developing AMD (primary prevention) (Table 3). Guidelines from Canada [29], Germany [34], and Australia [31] included recommendations on the value of dietary modification for primary prevention. With regard to supplements, a guideline produced by an expert panel of optometrists in the United States [28] recommended the use of xanthophil supplements (lutein, zeaxanthin, and mesozeaxanthin) in 'sub-clinical' AMD and another suggested that supplementation with antioxidants may be beneficial for those who are nutritionally deficient [26]. Five guidelines specifically stated that there was evidence that use of high- dose anti-oxidant vitamin and mineral supplements was not beneficial for primary prevention (Table 3) [24,27,31,34,35].

All of the included guidelines provided recommendations regarding nutritional strategies for secondary prevention (i.e., slowing the progression of AMD) (Table 4). Five guidelines [24,28,30,31,33] included dietary advice, consisting of encouraging people with AMD to eat a healthy diet and, in particular, to increase consumption of foods rich in the macular carotenoids (lutein and zeaxanthin) and/or eat more oily fish as a source of omega-3 essential fatty acids. With regard to nutritional supplements, all guidelines addressed the topic of antioxidant vitamin and mineral supplements; the vast majority referred to supplements containing the combinations of antioxidant vitamins, zinc and carotenoids used in the Age-Related Eye Disease Studies (AREDS) or AREDS2 studies [7,8]. Only the U.K. National Institute for Health and Care Excellence (NICE) guideline [25] did not advocate the use high-dose antioxidant vitamin and mineral supplements for the secondary prevention of AMD. The NICE guideline committee concluded that "the current clinical evidence was not able to demonstrate a clear treatment benefit of antioxidant vitamin and mineral supplement for people with early AMD and was insufficient to make a strong recommendation on the use of these supplements". The majority of guidelines acknowledged the risks as well as the benefits of antioxidant vitamin and mineral supplementation. Although acknowledging the lack of evidence, two guidelines $[28,34]$ stated that omega-3 fatty acid supplements could be considered. By contrast, the NICE committee [25] concluded that "omega-3 fatty acid supplementation had no meaningful effect on AMD progression and visual acuity, and that therefore no recommendations could be made on this topic". 


\subsection{Clinical Guideline Quality Scores}

The overall inter-rater agreement on scoring of the individual items was excellent (correlation coefficient: 0.81 (95\% confidence interval (CI) 0.76 to 0.84$)$ ).

The quality of each guideline, across each of the AGREE II domains, is shown in Table 5. The median percentage scores (\%) for different domains ranged from $13 \%$ to $75 \%$, with substantial heterogeneity in quality between guidelines. Only two of six domains had a median score over $50 \%$ ('Scope and Purpose' and 'Clarity of presentation'). The lowest scoring domains were 'Applicability' (median 13\% (range 0 to 98\%)), 'Editorial Dependence' (median 20.8\% (range 0 to $100 \%$ )), and Rigour of Development (median 20.4\% (range 9.2 to 95.9\%)). For the 'Rigour of Development' domain, only four of the 13 guidelines achieved a score $\geq 50 \%$; this was largely due to a failure to demonstrate that systematic methods had been used to search for relevant evidence, no description of the criteria used for selecting and/or evaluating the evidence, and no information on the methods used by the developers to formulate recommendations.

\subsection{Mapping Clinical Guideline Recommendations to Systematic Review Evidence}

We extracted individual recommendations relating to nutritional strategies for both preventing and slowing the progression of AMD (see Tables 3 and 4 for an overview). For some guidelines, no evidence was provided to support nutritional recommendations [33,36]. For other guidelines, individual studies (i.e., RCTs or prospective cohort studies) were cited. Only four guidelines $[24,25,30,34]$ included evidence from systematic reviews despite the availability of reliable systematic reviews for all of the extracted nutritional recommendations [13-23]. 
Table 3. Nutritional recommendations for primary prevention of age-related macular degeneration (AMD).

\begin{tabular}{|c|c|c|c|c|c|}
\hline Clinical Guideline & Dietary Advice & $\begin{array}{l}\text { Use of Antioxidant or } \\
\text { Mineral Supplements }\end{array}$ & $\begin{array}{l}\text { Use of Omega-3 Fatty } \\
\text { Acid Supplements }\end{array}$ & $\begin{array}{l}\text { Contraindications or } \\
\text { Side Effects of } \\
\text { Supplements }\end{array}$ & $\begin{array}{l}\text { Systematic Review Cited } \\
\text { with Recommendation }\end{array}$ \\
\hline American Optical Association 2004 & NR & $\checkmark^{4}$ & NR & $\checkmark$ & None \\
\hline International Council of Ophthalmology 2007 & NR & NR & NR & N/A & N/A \\
\hline Spanish Retina and Vitreous Society 2009 & NR & NR & NR & N/A & N/A \\
\hline Canadian Expert Consensus 2012 & NR & NR & NR & N/A & N/A \\
\hline German Ophthalmological Society 2014 & $\checkmark 1$ & $\checkmark^{5}$ & NR & $\mathrm{N} / \mathrm{A}$ & Yes \\
\hline Eye Health Council of Ontario (Canada) 2015 & $\checkmark^{2}$ & $\checkmark^{5}$ & NR & $\mathrm{N} / \mathrm{A}$ & None \\
\hline National Health Committee (New Zealand) 2015 & NR & NR & NR & $\mathrm{N} / \mathrm{A}$ & $\mathrm{N} / \mathrm{A}$ \\
\hline American Academy of Ophthalmology 2015 & NR & $\checkmark^{5}$ & NR & N/A & None \\
\hline Vitreo-Retinal Society of the Philippines 2016 & NR & $\checkmark^{5}$ & NR & N/A & None \\
\hline NICE Clinical Knowledge Summary (CKS) 2016 & NR & $\checkmark^{5}$ & NR & N/A & Yes \\
\hline Clinical Advisory Committee (United States) 2017 & NR & $\checkmark^{6}$ & NR & NR & None \\
\hline NICE Guideline (NG82) 2018 & NR & NR & NR & N/A & $\mathrm{N} / \mathrm{A}$ \\
\hline Optometry Australia 2019 & $\checkmark^{3}$ & $\checkmark^{7}$ & NR & NR & None \\
\hline
\end{tabular}

Abbreviations: $\checkmark$, recommendation included; NICE, National Institute for Health and Care Excellence; NR, no recommendation. Guideline recommendations: ${ }^{1}$ Balanced diet; ${ }^{2}$ A diet high in green, leafy vegetables (rich in antioxidants and carotenoids); ${ }^{3}$ A diet high in macular carotenoids (zeaxanthin and lutein) and omega-3 fatty acids; ${ }^{4}$ Antioxidant nutrient

supplements (particularly for nutritionally deficient); ${ }^{5}$ Evidence of no benefit for antioxidant vitamin and/or mineral supplements for primary prevention; ${ }^{6}$ Supplement containing xanthophylls (lutein, zeaxanthin, meso-zeaxanthin) for 'sub-clinical' AMD. ${ }^{7}$ Supplements not currently recommended for people with normal ageing changes. 
Table 4. Nutritional recommendations for secondary prevention (i.e., slowing the progression) of age-related macular degeneration (AMD).

\begin{tabular}{|c|c|c|c|c|c|}
\hline Clinical Guideline & Dietary Advice & $\begin{array}{l}\text { Use of Antioxidant or } \\
\text { Mineral Supplements }\end{array}$ & $\begin{array}{l}\text { Use of Omega-3 Fatty } \\
\text { Acid Supplements }\end{array}$ & $\begin{array}{l}\text { Contraindications or } \\
\text { Side Effects of } \\
\text { Supplements }\end{array}$ & $\begin{array}{l}\text { Systematic Review Cited } \\
\text { with a Recommendation }\end{array}$ \\
\hline American Optical Association 2004 & NR & $\checkmark^{6}$ & NR & $\checkmark$ & None \\
\hline International Council of Ophthalmology 2007 & NR & $\checkmark 7 \mathrm{a}$ & NR & NR & None \\
\hline Spanish Retina and Vitreous Society 2009 & $\checkmark 1$ & $\checkmark 7 \mathrm{a}$ & NR & $\checkmark$ & None \\
\hline Canadian Expert Consensus 2012 & $\checkmark^{2}$ & $\checkmark 7 a$ & NR & $\checkmark$ & Yes \\
\hline German Ophthalmological Society 2014 & NR & $\checkmark 7 \mathrm{a}$ & $\checkmark 10$ & $\checkmark$ & Yes \\
\hline Eye Health Council of Ontario (Canada) 2015 & $\checkmark^{3}$ & $\checkmark 7 a$ & $\checkmark 10$ & $\checkmark$ & None \\
\hline National Health Committee (New Zealand) 2015 & NR & $\checkmark 7 \mathrm{a}$ & NR & NR & None \\
\hline American Academy of Ophthalmology 2015 & NR & $\checkmark 7 \mathrm{a}$ & NR & $\checkmark$ & None \\
\hline Vitreo-Retina Society of the Philippines 2016 & NR & $\checkmark 7 \mathrm{a}$ & NR & $\checkmark$ & None \\
\hline NICE Clinical Knowledge Summary (CKS) 2016 & $\checkmark^{3}$ & $\checkmark 7 \mathrm{a}$ & NR & $\checkmark$ & Yes \\
\hline Clinical Advisory Committee (United States) 2017 & $\checkmark^{4}$ & $\checkmark 7 a$ & $\checkmark 9$ & NR & None \\
\hline NICE Guideline (NG82) 2018 & NR & $\checkmark^{8}$ & $\checkmark 10$ & $\checkmark$ & Yes \\
\hline Optometry Australia 2019 & $\checkmark^{5}$ & $\checkmark^{7 b}$ & NR & NR & None \\
\hline
\end{tabular}

Abbreviations: $\checkmark$, recommendation included; NICE, National Institute for Health and Care Excellence; NR, no recommendation. Guideline recommendations: ${ }^{1}$ Diet rich in carotenoids (lutein and zeaxanthin) and omega-3 fatty acids; ${ }^{2}$ Dietary intake of antioxidants, docosahexaenoic acid, and omega-3 fatty acids; ${ }^{3}$ Diet high in fresh fruit and green, leafy vegetables (rich in antioxidants and carotenoids): ${ }^{4}$ Consume oily fish rich in DHA and follow healthier eating styles e.g., Mediterranean diet; ${ }^{5}$ A diet rich in green leafy vegetables, fish and antioxidants should be encouraged; ${ }^{6}$ Antioxidant nutrient supplements; ${ }^{7 a}$ Age-Related Eye Disease Studies (AREDS) or AREDS2 supplement recommended; ${ }^{\text {tb }}$ AREDS or AREDS2 supplement may be beneficial, and should be discussed in conjunction with the patient's general medical practitioner. ${ }^{8}$ No evidence that benefit of AREDS supplement outweighs the risk; ${ }^{9}$ Omega-3 fatty acid supplement recommended; ${ }^{10}$ No evidence for benefit of omega-3 fatty acid supplements. 
Table 5. Quality, measured in \%, of the included AMD clinical guidelines, based on the six assessment domains of the Appraisal of Guidelines for Research and Evaluation II (AGREE II) appraisal tool. The scores for each of the six domains were calculated by summing up all the scores of the individual items for that domain, and then scaling the total as a percentage of the maximum possible score for each domain.

\begin{tabular}{|c|c|c|c|c|c|c|}
\hline \multirow[b]{2}{*}{ Clinical Guideline } & \multicolumn{6}{|c|}{ AGREE II Domains (\%) } \\
\hline & $\begin{array}{l}\text { Scope and } \\
\text { Purpose }\end{array}$ & $\begin{array}{l}\text { Stakeholder } \\
\text { Involvement }\end{array}$ & $\begin{array}{c}\text { Rigour of } \\
\text { Development }\end{array}$ & $\begin{array}{c}\text { Clarity of } \\
\text { Presentation }\end{array}$ & Applicability & $\begin{array}{c}\text { Editorial } \\
\text { Independence }\end{array}$ \\
\hline American Optical Association 2004 & 52.8 & 30.6 & 9.2 & 30.6 & 10.9 & 87.5 \\
\hline International Council of Ophthalmology 2007 & 58.3 & 30.6 & 10.2 & 58.3 & 2.2 & 0.0 \\
\hline Spanish Retina and Vitreous Society 2009 & 63.9 & 41.7 & 21.4 & 36.1 & 13.0 & 0.0 \\
\hline Canadian Expert Consensus 2012 & 75.0 & 47.2 & 36.7 & 75.0 & 28.3 & 20.8 \\
\hline German Ophthalmological Society 2014 & 30.6 & 25.0 & 19.4 & 52.8 & 0.0 & 0.0 \\
\hline Eye Health Council of Ontario (Canada) 2015 & 61.1 & 22.2 & 14.3 & 41.7 & 13.0 & 20.8 \\
\hline National Health Committee (New Zealand) 2015 & 75.0 & 13.9 & 20.4 & 55.6 & 30.4 & 0.0 \\
\hline American Academy of Ophthalmology 2015 & 83.3 & 63.9 & 72.4 & 94.4 & 17.4 & 75.0 \\
\hline Vitreo-Retina Society of the Philippines 2016 & 83.3 & 44.4 & 56.1 & 88.9 & 13.0 & 45.8 \\
\hline NICE Clinical Knowledge Summary (CKS) 2016 & 88.9 & 83.3 & 68.4 & 91.7 & 39.1 & 87.5 \\
\hline Clinical Advisory Committee (United States) 2017 & 52.8 & 22.2 & 9.2 & 44.4 & 4.3 & 0.0 \\
\hline NICE Guideline (NG82) 2018 & 94.4 & 88.9 & 95.9 & 100.0 & 97.8 & 100.0 \\
\hline Optometry Australia 2019 & 83.3 & 41.7 & 19.4 & 75.0 & 19.6 & 0.0 \\
\hline Median (range) & $\begin{array}{c}75.0 \\
\text { (range } 30.6 \text { to } \\
94.4 \% \text { ) }\end{array}$ & $\begin{array}{c}41.7 \\
\text { (range } 13.9 \text { to } \\
88.9 \% \text { ) }\end{array}$ & $\begin{array}{c}20.4 \\
\text { (range } 9.2 \text { to } \\
95.9)\end{array}$ & $\begin{array}{c}58.3 \\
\text { (range } 30.6 \text { to } \\
100 \% \text { ) }\end{array}$ & $\begin{array}{c}13.0 \\
\text { (range } 2.2 \text { to } \\
97.8 \% \text { ) }\end{array}$ & $\begin{array}{c}20.8 \\
\text { (range } 0 \text { to } \\
100 \% \text { ) }\end{array}$ \\
\hline
\end{tabular}




\section{Discussion}

Nutritional supplements for 'eye health' are marketed at the general population, and are also widely recommended by optometrists and ophthalmologists for people who have clinical signs of AMD [37,38]. Clinical practice guidelines are a useful method for presenting evidence-based recommendations to health care professionals, and are intended to be used to inform clinical decision-making and reduce potential variations in practice. The aims of the current study were to identify all clinical guidelines for AMD that include recommendations relating to diet and/or nutrition; to evaluate the methodological quality of these guidelines using the AGREE II tool (the most widely used instrument for appraising clinical practice guidelines); and to investigate whether specific nutritional recommendations were underpinned by evidence from systematic reviews.

We identified 13 clinical practice guidelines meeting our inclusion criteria. The overall quality of these guidelines was judged to be low to moderate, with the median percentage scores for four of the six AGREE II quality domains being below $50 \%$. The lowest scoring domains were 'Rigour of Development', 'Applicability', and 'Editorial Independence'. Low scores in these domains are of major concern, as they relate to: the process by which relevant research evidence is gathered and synthesised; the methods used to formulate the recommendations; the likely barriers and facilitators to implementation of the guideline recommendations; and how conflicts of interest are managed. It is important that guideline users are able to identify the evidence underpinning each recommendation, however many of the guidelines failed to make an explicit link to the evidence used to formulate recommendations. Transparency with respect to the reporting of conflicts of interest amongst guideline development panels is also essential, to avoid the potential for biased recommendations. Patient engagement in the development of the clinical practice guidelines was also generally poor, despite standards (including those defined by the World Health Organisation) [39] recommending the inclusion of patients on guideline development panels. The intent of this engagement is to ensure a focus on patient-centred guidelines that can enhance the quality of care [40].

Only one guideline (the National Institute for Health and Care Excellence (NICE) guideline NG82) achieved high scores in all of the AGREE II assessment domains. This is perhaps unsurprising, since the NICE guideline development manual states that NICE guidelines "are based on internationally accepted criteria of quality as detailed in the Appraisal of Guidelines of Research and Evaluation II (AGREE II) instrument". The development and implementation of high quality clinical practice guidelines requires substantial time, expertise and resources. Less formal guideline development groups, such as those produced by 'expert panels' or professional organisations, may lack the resources and/or methodological expertise to produce guidelines of the highest quality. In such circumstances, adapting existing high quality guidelines to local contexts may be an alternative to de novo development. The AMD Preferred Practice Patterns (PPP) Philippines (2016) [35] is an example of where this strategy has been adopted. This guideline was prepared by a panel from the Vitreo-Retina Society of the Philippines for the Philippine Academy of Ophthalmology, by adapting recommendations from the American Academy of Ophthalmology (AAO) PPP for AMD (updated in 2015).

Many robust systematic reviews have considered the merit of a variety of dietary and nutritional recommendations for AMD. In the present study, we used the systematic evidence searches performed by Lindsley et al. [11] and Downie et al. [12] to identify relevant systematic reviews, up to March 2017. These studies identified 11 unique systematic reviews on nutritional interventions for AMD, published between 2007 and 2016. Four of these reviews were published in the Cochrane Library. Mapping of clinical practice guideline recommendations to systematic review evidence showed that four of the included guidelines $[24,25,30,34]$ made reference to systematic reviews to support their nutritional recommendations. Other guidelines included evidence from individual RCTs or non-randomised trials only. In some cases, recommendations were provided with no supporting citations. Although it is likely that a proportion of the systematic review evidence would not have been available to the panels developing the three earliest guidelines, published between 2004-2009 [26,33,36], this evidence would certainly have been available to later panels and could have been used to inform their recommendations. 
Inconsistent uptake of evidence from systematic reviews by decision-makers and guideline developers is known to be related to several factors, including lack of awareness, lack of access and lack of familiarity [41]. The outcome of failing to use the best-available research evidence (i.e., systematic reviews) to inform practice guidelines is that this time-intensive, rigorous research effort is wasted. Furthermore, the entire rationale for the guideline to support evidence-based practice, and optimise patient outcomes is potentially not achieved.

Of the nine guidelines that considered nutritional approaches for the primary prevention of AMD, most considered the potential merit of dietary modification, although some also made recommendations in relation to nutritional supplementation. Several epidemiological studies have investigated whether specific dietary patterns and/or foods are associated with a reduced risk of developing AMD. A meta-analysis of prospective cohort, case-control and cross-sectional studies concluded that consumption of two or more servings of oily fish per week was beneficial in the primary prevention of AMD [13]. Another recent systematic review reported that a high consumption of vegetables rich in carotenoids and oily fish containing omega-3 fatty acids was beneficial for those at risk of AMD [42]. However, emphasising the potential difference in nutritional benefit(s) derived from whole foods versus supplementation, consuming anti-oxidant supplements does not prevent the primary onset of AMD [16]. It is therefore concerning that a recent (2017) U.S. 'guideline' [28], produced by a panel of optometrists, recommended prescribing xanthophil supplements (i.e., lutein, zeaxanthin and mesozeaxanthin) to patients with 'sub-clinical' AMD, as "it is better to prescribe a supplement than not to prescribe a supplement". Although termed a 'guideline', this document achieved the poorest quality scoring of all those included, with characteristics of a 'clinical viewpoint' rather than a 'guideline' per se.

Nutritional strategies for secondary prevention of AMD (i.e., slowing progression of the disease) were included in all of the clinical guidelines. Five guidelines $[24,28,33,34]$ included dietary advice, consisting of recommendations for people with AMD to eat a healthy balanced diet and, specifically to increase the consumption of foods rich in the macular carotenoids and/or eat more fish (as a source of omega-3 fatty acids). A Mediterranean diet has been linked to a reduced risk of AMD progression [43]. Epidemiological studies suggest that a high dietary intake of omega-3 fatty acids is associated with a significant reduction in the risk of both intermediate [44,45] and late-stage AMD [46,47]. However, the best-available research evidence does not support long-chain omega-3 fatty acid supplementation for slowing disease progression [15]. Despite this, and acknowledging that there is a lack of evidence to substantiate such a position, two recent clinical guidelines $[28,34]$ included a recommendation for clinicians to consider this approach.

With respect to high-dose anti-oxidant vitamin and mineral supplements for managing AMD, there were divergent recommendations. Notably, only the U.K. NICE guideline [25] did not advocate prescribing supplements containing the formulation of antioxidant vitamins, zinc and carotenoids investigated in the Age-Related Eye Disease Studies (AREDS [7] and AREDS2 [8]). This recommendation was based upon the committee's assessment of the limitations of the current, best-available evidence, and a need for "further research in this area". Indeed, there remain several key unanswered questions. For example, the minimum effective dose required for a given antioxidant to impart a potential retinoprotective effect remains unclear. Whether a single component, or a combination of components, represents the optimal formulation is also uncertain. The NICE guideline committee recommended "a well conducted randomised trial [ ... ] to provide an evidence base for the benefits and risks of individual components of the antioxidant supplements, and provide the ability to establish the treatment effect of antioxidant supplementation (the AREDS2 formula) on AMD progression by comparing AREDS2 formula with no treatment (for instance normal diet)". Although many of the guidelines considered the potential contraindications and/or side effects of high-dose antioxidant vitamin and mineral supplements, four of the guidelines did not articulate these risks $[28,31,32,36]$. Given that the decision to prescribe an intervention should balance the potential risks versus benefits of treatment, lack of this key information to guide clinical decision-making is a major shortcoming. 
A key strength of the current study was the comprehensive search to identify eligible clinical practice guidelines. Data extraction and quality appraisal were conducted independently by two reviewers, using a recognised assessment tool (AGREE II), which has established metrics of reliability and validity [48]. Overall, the reporting of methodological details for clinical guideline development was poor and therefore it was not possible to make a judgement as to whether the guidelines had used an appropriate evidence-based approach in their development.

\section{Conclusions}

Despite the availability of robust systematic reviews evaluating the efficacy and safety of nutritional interventions for AMD, this study found evidence that these resources are infrequently used to support recommendations in AMD clinical practice guidelines. Consequently, guidelines often present conflicting recommendations that could lead to variations in clinical care. The AGREE II quality evaluations of the included guidelines identified several key areas that require improvement, particularly the rigour of development, managing potential conflicts of interests, and presenting strategies for implementing guideline recommendations into daily practice.

Supplementary Materials: The following are available online at http://www.mdpi.com/2072-6643/11/4/823/s1, File S1: Search Strategy.

Author Contributions: J.G.L., J.R.E. and L.E.D. were involved in all aspects of the study conception and design, data acquisition, analysis and interpretation. J.G.L. wrote the first draft of the manuscript and this was critically revised by J.R.E. and L.E.D. All authors approved the final version of the manuscript.

Conflicts of Interest: The authors declare no conflict of interest in relation to this work.

\section{References}

1. Coleman, H.R.; Chan, C.C.; Ferris, F.L., 3rd; Chew, E.Y. Age-related macular degeneration. Lancet 2008, 372, 1835-1845. [CrossRef]

2. Bhutto, I.; Lutty, G. Understanding age-related macular degeneration (AMD): Relationships between the photoreceptor/retinal pigment epithelium/Bruch's membrane/choriocapillaris complex. Mol. Asp. Med. 2012, 33, 295-317. [CrossRef]

3. Mitchell, J.; Bradley, C. Quality of life in age-related macular degeneration: A review of the literature. Health Qual. Life Outcomes 2006, 4, 97. [CrossRef] [PubMed]

4. Srinivasan, S.; Swaminathan, G.; Kulothungan, V.; Raman, R.; Sharma, T.; Medscape. Prevalence and the risk factors for visual impairment in age-related macular degeneration. Eye 2017, 31, 846-855. [CrossRef]

5. Downie, L.E.; Keller, P.R. Nutrition and age-related macular degeneration: Research evidence in practice. Optom. Vis. Sci. 2014, 91, 821-831. [CrossRef]

6. Gorusupudi, A.; Nelson, K.; Bernstein, P.S. The Age-Related Eye Disease 2 Study: Micronutrients in the Treatment of Macular Degeneration. Adv. Nutr. 2017, 8, 40-53. [CrossRef] [PubMed]

7. Age-Related Eye Disease Study Research Group. A randomized, placebo-controlled, clinical trial of high-dose supplementation with vitamins $\mathrm{C}$ and $\mathrm{E}$, beta carotene, and zinc for age-related macular degeneration and vision loss: AREDS report no. 8. Arch. Ophthalmol. 2001, 119, 1417-1436. [CrossRef]

8. Age-Related Eye Disease Study 2 Research Group. Lutein + zeaxanthin and omega-3 fatty acids for age-related macular degeneration: The Age-Related Eye Disease Study 2 (AREDS2) randomized clinical trial. JAMA 2013, 309, 2005-2015. [CrossRef]

9. Institute of Medicine (US) Committee on Standards for Developing Trustworthy Clinical Practice Guidelines. Clinical Practice Guidelines We Can Trust; Graham, R.M.M., Wolman, D.M., Greenfield, S., Steinberg, E., Eds.; The National Academies Press: Washington, DC, USA, 2011; p. 290.

10. Wormald, R.; Evans, J. What Makes Systematic Reviews Systematic and Why are They the Highest Level of Evidence? Ophthalmic Epidemiol. 2018, 25, 27-30. [CrossRef]

11. Lindsley, K.; Li, T.; Ssemanda, E.; Virgili, G.; Dickersin, K. Interventions for Age-Related Macular Degeneration: Are Practice Guidelines Based on Systematic Reviews? Ophthalmology 2016, 123, 884-897. [CrossRef] 
12. Downie, L.E.; Makrai, E.; Bonggotgetsakul, Y.; Dirito, L.J.; Kristo, K.; Pham, M.N.; You, M.; Verspoor, K.; Pianta, M.J. Appraising the Quality of Systematic Reviews for Age-Related Macular Degeneration Interventions: A Systematic Review. JAMA Ophthalmol. 2018, 136, 1051-1061. [CrossRef] [PubMed]

13. Chong, E.W.; Wong, T.Y.; Kreis, A.J.; Simpson, J.A.; Guymer, R.H. Dietary antioxidants and primary prevention of age related macular degeneration: Systematic review and meta-analysis. BMJ 2007, 335, 755. [CrossRef]

14. Evans, J. Antioxidant supplements to prevent or slow down the progression of AMD: A systematic review and meta-analysis. Eye 2008, 22, 751-760. [CrossRef] [PubMed]

15. Lawrenson, J.G.; Evans, J.R. Omega 3 fatty acids for preventing or slowing the progression of age-related macular degeneration. Cochrane Database Syst. Rev. 2012, 11, CD010015. [CrossRef] [PubMed]

16. Evans, J.R.; Lawrenson, J.G. Antioxidant vitamin and mineral supplements for preventing age-related macular degeneration. Cochrane Database Syst. Rev. 2012, CD000253. [CrossRef]

17. Hodge, W.G.; Barnes, D.; Schachter, H.M.; Pan, Y.I.; Lowcock, E.C.; Zhang, L.; Sampson, M.; Morrison, A.; Tran, K.; Miguelez, M.; et al. Evidence for the effect of omega-3 fatty acids on progression of age-related macular degeneration: A systematic review. Retina 2007, 27, 216-221. [CrossRef]

18. Ma, L.; Liu, R.; Du, J.H.; Liu, T.; Wu, S.S.; Liu, X.H. Lutein, Zeaxanthin and Meso-zeaxanthin Supplementation Associated with Macular Pigment Optical Density. Nutrients 2016, 8, 426. [CrossRef] [PubMed]

19. Sin, H.P.; Liu, D.T.; Lam, D.S. Lifestyle modification, nutritional and vitamins supplements for age-related macular degeneration. Acta Ophthalmol. 2013, 91, 6-11. [CrossRef] [PubMed]

20. Vishwanathan, R.; Chung, M.; Johnson, E.J. A systematic review on zinc for the prevention and treatment of age-related macular degeneration. Investig. Ophthalmol. Vis. Sci. 2013, 54, 3985-3998. [CrossRef]

21. Wang, X.; Jiang, C.; Zhang, Y.; Gong, Y.; Chen, X.; Zhang, M. Role of lutein supplementation in the management of age-related macular degeneration: Meta-analysis of randomized controlled trials. Ophthalmic Res. 2014, 52, 198-205. [CrossRef]

22. Evans, J.R. Ginkgo biloba extract for age-related macular degeneration. Cochrane Database Syst. Rev. 2013, CD001775. [CrossRef] [PubMed]

23. Evans, J.R.; Lawrenson, J.G. Antioxidant vitamin and mineral supplements for slowing the progression of age-related macular degeneration. Cochrane Database Syst. Rev. 2012, 11, CD000254. [CrossRef] [PubMed]

24. Anonymous. Clinical Knowledge Summaries. Macular Degeneration-Age-Related. Available online: https://cks.nice.org.uk/macular-degeneration-age-related (accessed on 25 March 2019).

25. Anonymous. Age-Related Macular Degeneration. NICE Guideline [NG82]. Available online: https: //www.nice.org.uk/guidance/ng82 (accessed on 25 March 2019).

26. Anonymous; American Optical Association. Optometric Clinical Practice Guideline. Care of the Patient with Age-Related Macular Degeneration (CPG6). Available online: https://www.aoa.org/optometrists/toolsand-resources/clinical-care-publications/clinical-practice-guidelines (accessed on 25 March 2019).

27. Anonymous. American Academy of Ophthalmology Preferred Practice Pattern. Age-Related Macular Degeneration. Available online: https://www.aao.org/preferred-practice-pattern/age-related-maculardegeneration-ppp-2015 (accessed on 25 March 2019).

28. Anonymous. Practical Guidelines for the Treatment of AMD. Available online: https://www. reviewofoptometry.com/publications/ro1017-practical-guidelines-for-the-treatment-of-amd (accessed on 25 March 2019).

29. Anonymous. Guidelines for the Collaborative Management of Persons with Age-Related Macular Degeneration by Health- and Eye-Care Professionals. Available online: https://opto.ca/sites/default/files/ resources/documents/cjo_journal_online_ehco_guidelines_eng_v2.pdf (accessed on 25 March 2019).

30. Cruess, A.F.; Berger, A.; Colleaux, K.; Greve, M.; Harvey, P.; Kertes, P.J.; Sheidow, T.; Tourville, E.; Williams, G.; Wong, D. Canadian expert consensus: Optimal treatment of neovascular age-related macular degeneration. Can. J. Ophthalmol. 2012, 47, 227-235. [CrossRef] [PubMed]

31. Optometry Australia. 2019 Clinical Practice Guide for the Diagnosis, Treatment and Management of Age-Related Macular Degeneration. Available online: http://www.optometry.org.au/media/1185775/amd_ clinical_practice_guide_-_2019_final_designed_v5.pdf (accessed on 25 March 2019).

32. Anonymous; National Health Committee. Age-Related Macular Degeneration. Available online: http://www.moh.govt.nz/notebook/nbbooks.nsf/0/1169E93BA82E0E3BCC257F7F0007E835/\$file/ 150620_age-related_macular_degeneration_t2_updated-june15.pdf (accessed on 25 March 2019). 
33. Ruiz-Moreno, J.M.; Arias-Barquet, L.; Armada-Maresca, F.; Boixadera-Espax, A.; Garcia-Layana, A.; Gomez-Ulla-de-Irazazabal, F.; Mones-Carilla, J.; Pinero-Bustamante, A.; Suarez-de-Figueroa, M.; Sociedad Española de Retina yVítreo. Guidelines of clinical practice of the SERV: Treatment of exudative age-related macular degeneration (AMD). Arch. Soc. Esp. Oftalmol. 2009, 84, 333-344. [PubMed]

34. Deutsche Ophthalmologische, G. Dietary supplements in age-related macular degeneration. Current observations of the German Ophthalmological Society, the German Retina Society and the Professional Association of German Ophthalmologists (as of October 2014). Klin. Mon. Augenheilkd. 2015, 232, 196-201. [CrossRef]

35. Anonymous. Age-Related Macular Degeneration (AMD). Preferred Practice Patterns (PPP). Philippines. Available online: http://pao.org.ph/standard/Age\%20Related\%20Mac\%20Degen.pdf (accessed on 25 March 2019).

36. Anonymous; International Council of Ophthalmology/International Federation of Ophthalmological Societies (ICO). International Clinical Guidelines. Age-Related Macular Degeneration (Management Recommendations). Available online: http://www.icoph.org/downloads/ICOARMDMa.pdf (accessed on 25 March 2019).

37. Downie, L.E.; Keller, P.R. The self-reported clinical practice behaviors of Australian optometrists as related to smoking, diet and nutritional supplementation. PLoS ONE 2015, 10, e0124533. [CrossRef]

38. Lawrenson, J.G.; Evans, J.R. Advice about diet and smoking for people with or at risk of age-related macular degeneration: A cross-sectional survey of eye care professionals in the UK. BMC Public Health 2013, 13, 564. [CrossRef] [PubMed]

39. Schünemann, H.J.; Fretheim, A.; Oxman, A.D. Improving the use of research evidence in guideline development: 10. Integrating values and consumer involvement. Health Res. Policy Syst. 2006, 4, 22. [CrossRef]

40. Armstrong, M.J.; Rueda, J.D.; Gronseth, G.S.; Mullins, C.D. Framework for enhancing clinical practice guidelines through continuous patient engagement. Health Expect. 2017, 20, 3-10. [CrossRef]

41. Wallace, J.; Nwosu, B.; Clarke, M. Barriers to the uptake of evidence from systematic reviews and meta-analyses: A systematic review of decision makers' perceptions. BMJ Open 2012, 2. [CrossRef]

42. Chapman, N.A.; Jacobs, R.J.; Braakhuis, A.J. Role of diet and food intake in age-related macular degeneration: A systematic review. Clin. Exp. Ophthalmol. 2019, 47, 106-127. [CrossRef] [PubMed]

43. Merle, B.M.J.; Colijn, J.M.; Cougnard-Gregoire, A.; de Koning-Backus, A.P.M.; Delyfer, M.N.; Kiefte-de Jong, J.C.; Meester-Smoor, M.; Feart, C.; Verzijden, T.; Samieri, C.; et al. Mediterranean Diet and Incidence of Advanced Age-Related Macular Degeneration: The EYE-RISK Consortium. Ophthalmology 2019, 126, 381-390. [CrossRef] [PubMed]

44. Christen, W.G.; Schaumberg, D.A.; Glynn, R.J.; Buring, J.E. Dietary omega-3 fatty acid and fish intake and incident age-related macular degeneration in women. Arch. Ophthalmol. 2011, 129, 921-929. [CrossRef]

45. Seddon, J.M.; George, S.; Rosner, B. Cigarette smoking, fish consumption, omega-3 fatty acid intake, and associations with age-related macular degeneration: The US Twin Study of Age-Related Macular Degeneration. Arch. Ophthalmol. 2006, 124, 995-1001. [CrossRef] [PubMed]

46. Chong, E.W.; Kreis, A.J.; Wong, T.Y.; Simpson, J.A.; Guymer, R.H. Dietary omega-3 fatty acid and fish intake in the primary prevention of age-related macular degeneration: A systematic review and meta-analysis. Arch. Ophthalmol. 2008, 126, 826-833. [CrossRef]

47. Sangiovanni, J.P.; Agron, E.; Meleth, A.D.; Reed, G.F.; Sperduto, R.D.; Clemons, T.E.; Chew, E.Y.; Age-Related Eye Disease Study Research Group. \{omega\}-3 Long-chain polyunsaturated fatty acid intake and 12-y incidence of neovascular age-related macular degeneration and central geographic atrophy: AREDS report 30, a prospective cohort study from the Age-Related Eye Disease Study. Am. J. Clin. Nutr. 2009, 90, 1601-1607. [CrossRef]

48. Brouwers, M.C.; Kho, M.E.; Browman, G.P.; Burgers, J.S.; Cluzeau, F.; Feder, G.; Fervers, B.; Graham, I.D.; Hanna, S.E.; Makarski, J.; et al. Development of the AGREE II, part 2: Assessment of validity of items and tools to support application. CMAJ 2010, 182, E472-E478. [CrossRef]

(C) 2019 by the authors. Licensee MDPI, Basel, Switzerland. This article is an open access article distributed under the terms and conditions of the Creative Commons Attribution (CC BY) license (http://creativecommons.org/licenses/by/4.0/). 